\title{
Model of integration in the prevention of psychoactive dependence during the study of anatomy
}

\begin{abstract}
In the event of a global approach to neuroanatomy and its place in the teaching programme during anatomy, this case will not have a consistent response and a panacea for the exact model of teaching. Neuroanatomy can be included in a spiral-like and teaching aiming to consistently further develop knowledge along the years of study, not to be included as an isolating topic. This study introduces a model for the prevention of psychoactive dependence and its integration in the field of neuroanatomy. The model is expressed in six phases.
\end{abstract}

Keywords: neuroanatomy, drug abuse, health models, prevention programme
Volume 6 Issue 3 - 202I

\author{
Maslarski I,' Ingilizova G² \\ 'Departmant of Anatomy, histology, pathology and forensic \\ medicine, University of Sofia, Bulgaria \\ ${ }^{2}$ Vita" Multidisciplinary Hospital for Active Treatment, Bulgaria
}

Correspondence: Ingilizova G,Vita" Multidisciplinary Hospital for Active Treatment, Sofia, Bulgaria, Tel +359887204480, ORCID 0000-0002-07I8-458I, Email ingilizova@mail.bg

Received: June 31, 202I | Published: July 15, 2021

\section{Introduction}

Health education and prevention programs are a common phenomenon, however this topic is widely misunderstood, more specifically how can health education and prevention be implemented. Health specialists in all fields have a very important role in the preparation of such programs. A large enthlasis is placed on psychologists and pedagogics, which do not have the necessary knowledge in relation of neuroanatomy foundation of these topics.

During the years we have witnessed the development of many different models, which have been part of the health education, models in relation with the planning and the study of the human anatomy have been no exception. During the planning stage of any educational programme, it is of primary importance to establish the correct choice of content. Such as the case in neuroanatomy, if studied in isolation and independently, the volume of neuroanatomy will seem far too strenuous for students, adding on top of learning about the connections that have to be made with other structures of the body, from which derive structures which get innervated. The study of certain mechanisms (for example the release of dopamine or the mechanism of action of cannabinoid receptors), without the regard of their primary application and topography, would be next to useless. Apart from the choice of information, planning of the programme and foundation of the theoretical information is not far behind in terms of importance, to make sure that high education effectiveness.

\section{Material and methods}

The experiment was conducted with two groups of first and second year respectively. They were experimentally trained in the model of drug abuse prevention outlined below. The students studying medicine and the experiment was performed on part of the curriculum in General Histology on the one hand and Anatomy on the other.

\section{Results}

This study introduces a model for the prevention of psychoactive dependence and its integration in the field in neuroanatomy. The model is expressed in six phases (Figure 1).

The first phase is introduction. This is characterised in the evaluation and establishment of target groups. Each academic year the University witnesses a wide variety of students in terms of character. Contact with students and discussion of the upcoming curriculum will follow. The verbal discussions and primary contact with the students lead to establishing the topics, which are recommended to be studied in addition to the compulsory programme of anatomy. These topics have the aim to prevent psychoactive dependence, an issue that is emphasised during the programme. ${ }^{1}$

The second phase of the model is based on the planning of the aims of the programme.

The third phase established the main problems and inconsistencies.

The fourth phase is the most difficult to implement. During the process of this type of programme or any similar programme for that matter, it is of prime importance to prepare a list containing the fundamental key concepts that relate to neuroanatomy and psychoactive dependence. In part to laydown in according to the present group of students, which topics will be discussed, how they will be presented and integrated according to the education programme. An important step in this phase is to establish a choice of criteria and indicators, through which the programme will be evaluate.

The fifth phase is in regard to the governing the activities which have been planned according to phases 1-4 with regard to neuroanatomy and the prevention of the abuse of psychoactive substance. This is a very specific step and it's necessary to plan the 
methods which will have a direct link with the directing stage of the process of teaching and the allocation of the activities to the students and between themselves and the teaching staff.
The sixth phase relates to specific grading of the results, relating to neuroanatomy knowledge, skills and transfer, in addition to the prevention of psychoactive dependence.

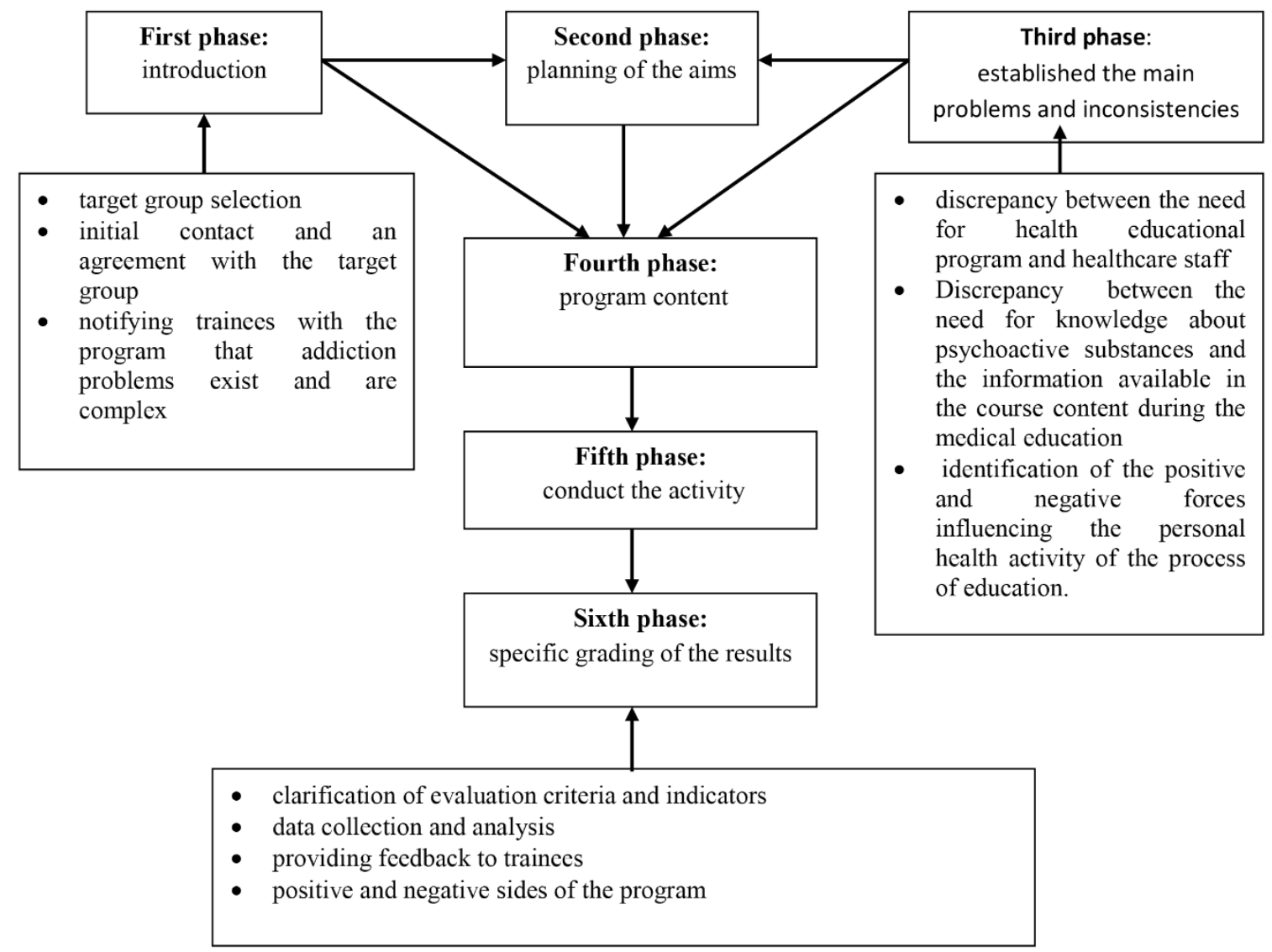

Figure I Model of planning of the prevention programme on drug addiction.

\section{Discussion}

In the event of a global approach to neuroanatomy and its place in the teaching programme during anatomy, this case will not have a consistent response and a panacea for the exact model of teaching. It is best for the neuroanatomy program to be integrated and to have a spiral-like character. An example in this respect is impossible to be given to the students, like a separated cycle or separated from the other chapters of anatomy. The foundations of neuroanatomy are first layed in the basic cytology course, histology and embryology. After which, during the study of internal organs is necessary to study their innervation, without knowing the exact location of terminal nucleuses in the brain or the direction of the nerves to the organ in question as well as the autonomic regulation. Then follows the teaching of the main components of the CNS and PNS in detail, along with a recap during the study of topographical anatomy and the place of nerves among themselves and other neighbouring structures. It is well apparent that this information is difficult to digest, this difficulty is also noticed during the different phases of building upon. This could be due to a lack of clarity, lack of knowledge, which will be present later on. ${ }^{2}$

Also lacking is a time slot where the information on these topics is consolidated and recapped, which has the potential of great use among the future doctors. The biggest difficulties more often than not arise during the understanding of the nervous system. For this reason, it is quite a common worldwide practice for neuroanatomy to be recapped as a separate component of study, for example; an elective discipline. After a review of the content of neuroanatomy in the world spectre it is apparent that neuroanatomy is present in 8 of the following components of the CNS: development of the central nervous system; spinal cord; brainstem; cerebellum; forebrain; brain ventricles and csf formation; blood vessels of the central nervous system; functional correlation of the central nervous system. It is becoming more and more apparent that psychoactive substances are not studied in the first few years in medicine, furthermore emphasis is placed upon neurology and pharmacology to establish a concept of their existing, mechanism of action and the fight against their abuse.

According to the presented theoretical model for prevention of psychoactive substances, based on the integration of neuroanatomy, during the study (Figure 1). It includes elements from the PRECEEDEPROCEED model of L. Green and M. Kreuter. ${ }^{3,4}$ PRECEEDE stands for Predisposing, Reinforcing and Enabling Constructs in Educational/ Environmental Diagnosis and Evaluation. PROCEED stands for Policy, Regulatory and Organizational Constructs in Educational and Environmental Development. During the planning stage of the model the following factors are given special notice: predisposing, activating and stimulating factors, which are very important for the correct structuring of the programme. Due to this reason the preceding information in the programme includes not only certain facts about 
the structure and the effect of abuse with psychoactive substance but their mechanism of action. Triggering factors cause an indirect influence on the result of the study programme.

Reinforcing factors influence via a variety of situations, problem solving skills and participation of discussions, in which the people that are being taught receive support from the lecturer or from the other students. Every student has the opportunity to compare their own behaviour to that of the others, thereby developing their own concept for a given problem. During the planning the stage of the programme the influence of positive, negative and uncharacteristic factors, which are part of the PEN-3., ${ }^{5,6}$ Very often a person under the influence of their environment may start believing that since they can stop abusing the so- called "light drugs", the exact same case would apply to the opioid originating drugs. As all such substances fall in the common umbrella term "drugs" or "narcotics".

\section{Conclusion}

Psychoactive substances, their mechanism and effects would be a good idea to be included in the preclinical study programme. For example, in the study programme of cytology, histology and embryology as well as human anatomy, is suitable the inclusion of information for the mechanism of action of some psychoactive substances. The models of Health Education can be used as an example for the development of topics outside the psychoactive dependence.

Neuroanatomy can be included in a spiral-like and teaching aiming to consistently further develop knowledge along the years of study, not to be included as an isolated topic. Further developing the information without going into the clinic. For example, including information about the dopamine pleasure chain, types of cannabinoid receptors, modulation and change in the activity and speed of conduction of a neuronal impulse, mediator reuptake in the presynaptic membrane, blocking of receptors in postsynaptic membrane, role of protein channels and pumps. The last concepts can be used as an example for the influence of psychoactive substances and why an addiction develops.

\section{Acknowledgments}

None.

\section{Funding}

None.

\section{Conflicts of interest}

None.

\section{References}

1. Cornwall J, Sabine H. Anatomy, Education, and Ethics in a Changing World. Anat Sci Educ. 2019;12(4):329-331.

2. Caplan I, Matthew D. Of discomfort and disagreement: Unclaimed bodies in anatomy laboratories at United States medical schools. Anat Sci Educ. 2019;12(4):360-369.

3. Airhihenbuwa C. Health and culture: Beyond the Western paradigm, CA: Sage, Thousand Oaks. USA. 1995:pp. 25-42.

4. Airhihenbuwa CJ, Webster JA. Culture and African Contexts of HIV/ AIDS prevention care, and support. Journal of Social Aspects of HIV/ AIDS Research Alliance. 2004;1(1):4-13.

5. Kreuter M, Green L. The new PRECEDE: A workshop for health education leaders. Helsinki, Finnish Council for Health Education. 1991;1;435-436.

6. Kreuter M. PATCH: Its origin, basic concepts, and links to contemporary public health policy. Journal of Health Education. 1992;23(3):135-139. 